\title{
Micropropagation of Temperate Cymbidium via Rhizome Culture
}

Kee Yoeup Paek ${ }^{1}$ and Toyoki Kozai ${ }^{2}$

Additional ImDeX words. asymbiotic, oriental, protocorm-like bodies, shoot tip

Sumмany. We report the results of serial studies aimed at clarifying several factors affecting organogenesis in rhizome culture of temperate Cymbidium species and their hybrids. The growth patterns and regeneration ability of rhizomes derived from asymbiotic seed or shoot tip culture vary according to media composition, kinds and concentrations of plant growth regulators, culture conditions, and species and varieties. $N^{\sigma}$ benzyladenine was the best cytokinin for inducing shoot formation, for switching rhizome tissues into protocorm-like bodies, and for directly forming multiple shoots from branched rhizomes. Activated charcoal appeared to be necessary for producing healthy plantlets and for stimulating shoot growth at levels of $0.1 \%$ to $0.3 \%$ but concomitantly decreased rhizome growth. Sucrose at $5 \%$ was the most effective concentration for shoot induction from rhizomes. The above results support the conclusion that organogenic pathways between tropical, subtropical, and temperate Cymbidium species may be controlled by the genetic backgrounds of the species or cultivars.

\section{Dypical terrestrial Cymbidium species are distributed in temper- ate eastern Asia including Korea, Japan, Taiwan, and China. 1 Cymbidium species found in tropical and subtropical Asian} countries, such as Nepal, the Philippines, Malaysia, Thailand, and Sri Lanka, are not regarded as temperate or oriental orchids. This is simply because many of the flowers of cymbidiums growing in tropical or subtropical Asian countries are very showy and colorful, which western people favor, but that have little or no fragrance or stripes on the leaves, which oriental people favor. Such a difference in terms of appreciation modes and value standards between oriental and western people seems to originate from traditional living customs and gardening culture. The origin of the value system in which people more highly regard fragrance and the several types of leaf chimera and shapes comes from China.

${ }^{1}$ Professor, Research Center for the Development of Advaneed Horticultural Technology, Chungbuk National University, Cheongju 361-763, Korea.

${ }^{2}$ Professor, Laboratory of Horticultural Engineering, Department of Bioproduction Science, Faculty of Horticulture, Chiba Univcrsity, Matsudo, Chiba 271, Japan.

This research was partially funded by SGRF/PTRP (Problem-Oriented Technology Development Project for Agriculture, Forestry, and Fisheries) in Korea. We extend thanks to G.B. Shim, who provided preserved materials for this project. We also thank S.H. Ma and B.M. Marisa for providing technical assistance. 
The historical background of Korea's native orchids stretches back as far as Europe's does; however, there are great differences between the principles of cultivation. Oriental Cymbidium cultivation in Korea appreciatcs the inter- and intraspecific variants of the indigenous species and natural hybrids. Collectors usually do not hybridize them, rather collect from the habitat, especially in the cases of C. kanran and C. goeringii. For Asiatic terrestrial cymbidiums, which are strongly endangered by cxtinction in their natural habitats, only some kinds of asymbiotic seed culture and a single asexual method of conventional division are available. Although strongly protected by the Nature Preservation Law, these plants are still being collected by so-called orchid lovers to bring them home for horticulture. Many habitats have been reduced and devastated. With the above mentioned considerations, our team has applied a tissue-culture system for micropropagation and in vitro breeding of tempcrate cymbidiums. However, these methods often suffer from relatively poor germination and a low frequency of caulogenesis from rhizome tissue and need sophisticated handling.

\section{In vitro approaches}

Under natural conditions, the multiplication rate of temperate and oriental cymbidiums, especially chimeral cymbidiums, which are very popular among orchid lovers, is very low compared to their subtropical or tropical counterparts. Generally the rate of newly formed shoots in vivo ranges between zero and three per plant per year, depending on the species and cultural practices. Especially, division of variegated cultivars is very limited. The introduction of an asymbiotic seed (Knudson, 1946) and shoot-tip culture (Morel, 1960) have had a profound influence on the methods by which orchids are propagated. Apart from the need for mass propagation of desired clones, tissuc-culture technique is used to free existing hybrids or clones from viral infections (Ishii, 1974). Indeed, it was in his attempt to produce virus-free cymbidiums from diseased plants that Morel (1960) developed the meristem culture method of multiplication and orchids became the first horticultural crop successfully mass-produced through tissue culture.

But eastern Asiatic temperate cymbidiums have received less attention. This is due in part to the rareness and difficulty involved in collecting sufficient amounts of experimental materials and the unimaginable prices caused by enthusiastic orchid lovers. At present, only a few reports (Chung et al., 1985; Hasegawa and Goi, 1987; Lee et al., 1986; Paek et al., 1987, 1989, 1990, 1990a, 1990b, 1993; Paek and Yeung, 1991;
Shimasaki and Uemoto 1990; Ucda and Torikata, 1969; Wang et al., 1988; Wu et al., 1987) exist in the literature concerning micropropagation of temperate cymbidiums.

Although tissue-culture techniques have been successfully applied since Morel's finding (1960) of meristem culture in Cymbidium in clonal propagation of orchids, recalcitrant species remain and there exist two distinctly different organogenetic pathways between temperate terrestrial and subtropical or tropical Cymbidium in vitro. In subtropical or tropical Cymbidium, organogenesis from asymbiotic seed or shoot-tip culture can be attained by way of a protocorm, but in temperate Cymbidium organogenesis is via the rhizome, which is recalcitrant to regeneration compared to the protocorm system. However, not much attention has been given to this matter.

The regeneration ability from rhizome culture can be controlled by manipulating environmental conditions or media supplemented with several additives or plant growth regulators. However, the principal organogenic capacity is governed by the genetic background of each species or variety.

The present work focused on several factors affecting organogenesis from rhizomes of temperate Cymbidium in vitro.

\section{Regeneration from rhizome culture}

EfFect of plant growth regulators. Usually, rhizomes of temperatc cymbidiums can be obtained from in vitro cultures of seeds, shoot tips, inflorescent stems, or flower organs. The growth patterns of rhizomes derived from cultured tissues in vitro are variable as affected by media composition, kinds and concentrations of plant growth regulators, various cultural conditions, and species and varieties. The growth patterns of rhizomes cultured on MS medium (Murashige and Skoog, 1962) are as follows: a) thick and long rhizomes with numerous branches (Fig. 1A); b) thin and long rhizomes with many branches (Fig. 1B); c) thick and short rhizomes with few branches (Fig. 1C); and d) thin and short rhizomes with many branches (Paek and Yeung, 1991). However, differentiation of rhizomes into plantlets does not occur easily and the cultural conditions needed for differentiation of the rhizomes into plantlets have not been fully investigated.

Several investigators have reported cytokinin-induced shoot formation in rhizome culture of temperate cymbidiums (Chung et al., 1985; Hasegawa and Goi, 1987; Paek et al., 1990). Usually, elongation and proliferation of rhizomes occurred in culture media supplemented with auxins. Cytokinins generally reduced rhizome growth based on an 


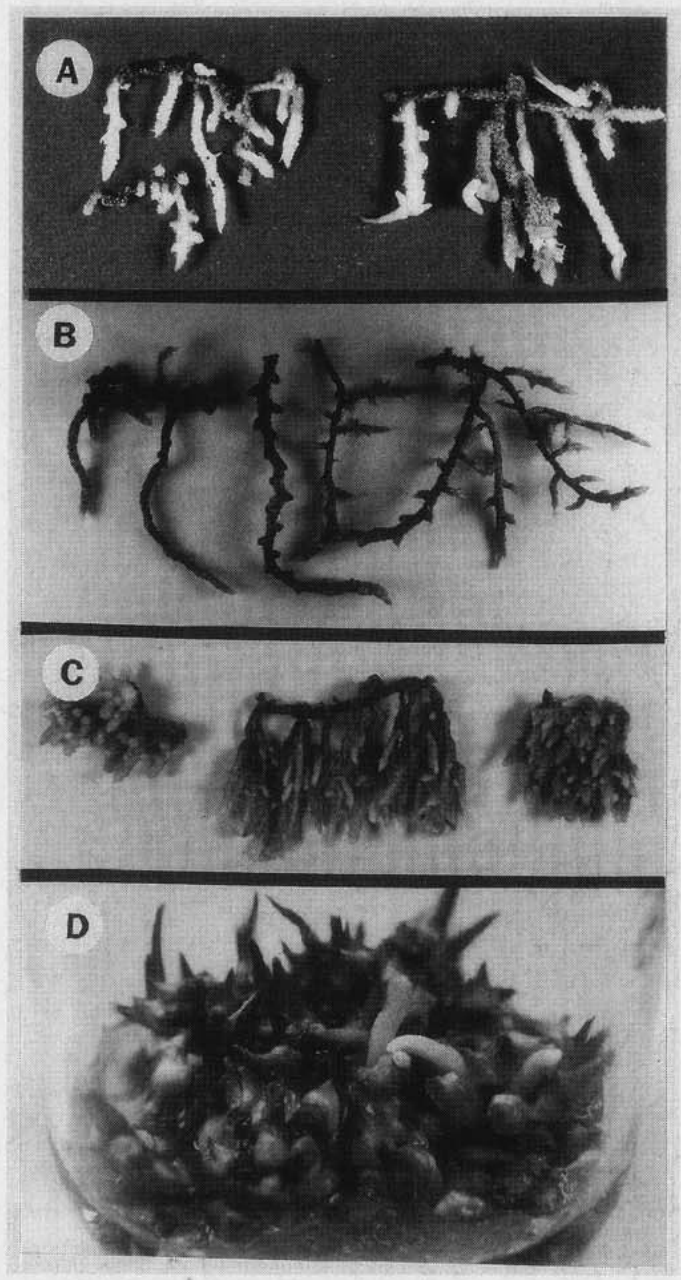

Fig. 1. Differential growth pattern of rhizomes as affected by Cymbidium species and growth regulators. (A) Thick and long rhizomes of C. neveo-magenatum; (B) thin and long rhizomes of C. kanran 'Namkuk'; (C) thick and short rhizomes of $C$. goeringii; cultured on MS medium containing $0.3 \mathrm{mg} / \mathrm{L}$ NAA; (D) protocorm-like bodies forming from rhizomes of $C$. kanvan cultured on MS medium supplemented with $5.0 \mathrm{mg} \cdot \mathrm{L}^{-1} \mathrm{BA}$ and $0.3 \mathrm{mg} \cdot \mathrm{L}^{-1} \mathrm{NAA}$.

increase in fresh weight and the number of branches. When rhizomes were cultured in the light, $N^{6}$-benzyladenine (BA) induced shoot formation and was the best cytokinin for switching rhizome tissues into protocormlike bodies and for forming multiple shoots directly from branched rhizomes (Fig. 1D) (Paek et al., 1989). Rhizomes cultured in the presence of $5.0 \mathrm{mg} \cdot \mathrm{L}^{-1} \mathrm{BA}$ rendered the medium yellow to brown in color.

We determined the polyphenol contents of rhizomes cultured in the high-level BAcontaining and control media. Total polyphenol contents reached maximum after 30 to 60 $\mathrm{d}$ in culture but decreased after $90 \mathrm{~d}$, and browning of the medium was also observed. When the extracts of rhizomes were analyzed using thin-layer chromatography, rhizomes cultured with BA showed more spots and medium browning than control (Paek et al., 1993).

Auxins clearly stimulated rhizome growth and increased the fresh weight of the explants. NAA, IBA, and IAA at $2 \mathrm{mg} \cdot \mathrm{L}^{-1}$ had similar effects; NAA and IBA were more effective than IAA. Rhizomes obtained from IBA treatment differed from those of the NAA treatment in that they were thinner in appearance and shoot formation was slightly inhibited upon transfer to the BA-containing medium (Paek and Yeung, 1991). Since NAA caused the highest fresh weight gain of the rhizome, it was used as the sole auxin in subsequent experiments (Fig. 2A).

BA treatment was important for multiple-shoot induction, subsequent growth of shoots, and prevented browning of medium when rhizome cultures were used. Pulsing treatment of rhizomes concentrations $>10$ $\mathrm{mg} \cdot \mathrm{L}^{-1}$ for $>10 \mathrm{~d}$ in the liquid shaker or in stationary culture was effective in producing protocorm-like bodies from rhizome tissue and multiple-shoot formation, while rhizome growth was strongly inhibited (Paek et al., 1990). The effect of the auxin to cytokinin ratio of the culture medium on organogenesis from rhizomes was also examined. Higher NAA $\left(2.0 \mathrm{mg} \cdot \mathrm{L}^{-1}\right)$ to $\mathrm{BA}\left(0.3 \mathrm{mg} \cdot \mathrm{L}^{-1}\right)$ ratios of the medium enhanced rapid growth of the cultured rhizomes. Lower NAA $\left(0.5 \mathrm{mg} \cdot \mathrm{L}^{-1}\right)$ to $\mathrm{BA}\left(5.0 \mathrm{mg} \cdot \mathrm{L}^{-1}\right)$ ratios in the culture medium resulted in the proliferation of numerous branches, formation of protocormlike bodies on the rhizomes, or both. These protocorm-like bodies developed poorly into plantlets and subsequent shoot growth was severely inhibited. The formation of shoots and growth of formed shoots from rhizomes were stimulated when the cultures were transferred to a medium of lower NAA to BA ratio after two or three passages in a medium of a higher NAA to BA ratio (Paek et al., 1989).

Structural investigations showed that the apical meristem responded differently to exogenously supplied plant growth regulators by reducing the cytoplasmic zone of the apical meristem and causing meristem derivatives to differentiate rapidly into vacuolated parenchyma cells. Leaf formation and development was retarded in the presence of auxin. BA-treated rhizomes had a well-defined meristem with several leaf primordia protecting the small dome-shaped apex. When rhizomes were grown in the auxin-containing medium, they enlarged in size and the surface of apex and the tip of rhizome had poorly developed leaf primordia (Paek et al., 1990a, 1990b).

It is difficult to determine the reason for the effects of each complex additive. Many have been tested with rhizomes, seeds, and other tissues, but there is little consistency in 
the results on rhizome growth and organogenesis. So far we have found that adding natural products such as homogenate of potato, tomato, banana, and apple into the medium helps prolong the culture of rhizomes for 4 to 5 months without subculture, and that the plantlets did not show symptoms of nutrient deficiency. Malt and yeast extracts had no effect in rhizome cultures on organogenesis. But the effect of several additives on organogenesis from rhizome culture depended on the kinds of medium, species, and cultivars. Natural products such as homogenate of banana, potato, and apple are usually used for temperate Cymbidium tissue culture (Paek et al., 1987). Potato homogenate was made of unsprouted potato tubers to prevent fermentation of the medium after culture.

Effect of activated charcoal. Charcoal generally reduced rhizome growth based on an increase in fresh weight but increased the number of branches of cultured rhizomes compared to the charcoal-free medium. Shoot

Fig. 2. Reestablishment of plantlets obtained by Cymbidium rhizome culture into orchid culture medium. (A) Rhizome proliferation from $1-\mathrm{cm}-$ long rhizome segments on MS medium containing 0.3 mg. $\mathrm{L}^{-1}$ NAA after 2 months in culture; (B) transferred proliferated rhizomes into $0.1 \%$ activated charcoal (AC) added medium (note shoot formation from the rhizome tips, which oriented into the medium); (C) vigorous shoot growth after 4 months culture on MS medium with 0.1 AC; (D) reestablishment of in vitro plantlets into fine bark after 6 months in culture of $C$. kanran and C. goeringii. formation was strongly inhibited in charcoal medium (Paek and Yeung, 1991). In the presence of activated charcoal, the tips of the rhizomes tended to grow into the medium. Charcoal appears to be necessary to produce healthy plantlets and stimulate shoot growth at concentrations of $0.1 \%$ to $0.3 \%$ after transferring formed shoots from a medium without charcoal to a medium containing charcoal (Fig. 2B). Generally charcoal can assist in adsorbing toxic substances that may be present in the media as a result of autoclaving or are produced by cultured tissues when first transferred to the medium or during further growth. It is also known to adsorb growth regulators added to the culture medium. In rhizome culture, the results indicate that the adsorption of cytokinins and auxins by activated charcoal reduces the effective growth regulator concentration required for shoot induction. A number of shoots of different Cymbidium species have been found to root readily in vitro when activated charcoal was added to a medium supplemented with auxin (Fig. 2C). Stimulation of root growth may be due partly to the fact that charcoal adsorbs inhibitory substances, adsorbs auxins, or excludes light from the medium.

EFfect of carbohydrates. Sugars as a carbon source for organogenesis from rhizome culture of temperate terrestrial Cymbidium species were not systematically evaluated. As a result, comparative studies were made that identified sugars and carbohydrates as capable or incapable of shoot formation and growth from rhizome culture. Species and cultivars vary in their organogenic ability on several sugars, but, in some in-

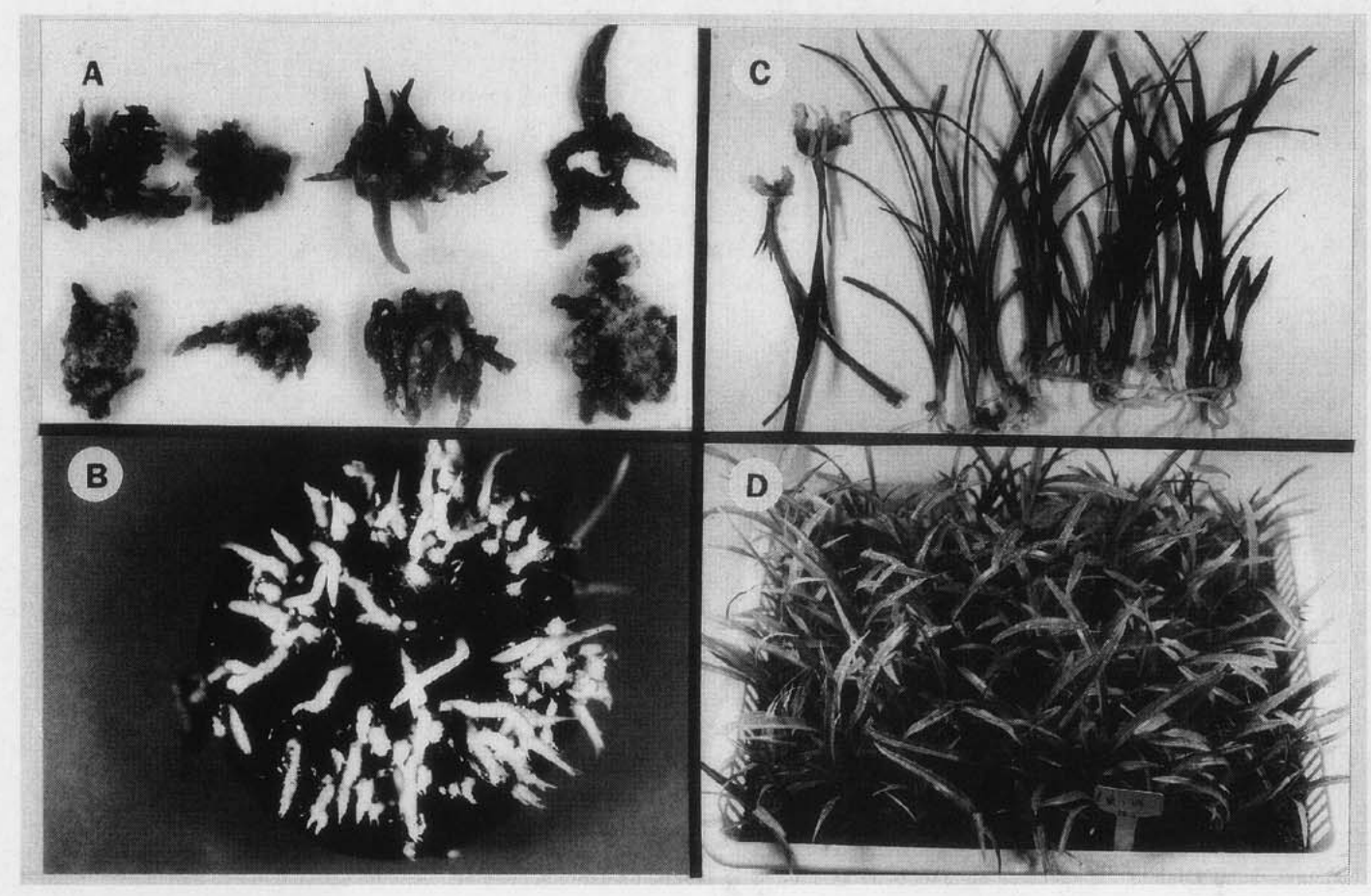


stances, the physiological problems are more complex than an appropriate carbon source (Arditti, 1977; Arditti and Ernst, 1993). Among the sugars tested, polysaccharides, other carbohydrates, and carboxylic acidsD-ribose, D-xylose, lactose, trehalose, Lrhamnose, starch, mannitol, sorbitol, malic acid, pyruvic acid, and succinic acid-were classified unsuitable as carbon sources for organogenesis from rhizome culturc. Cultured rhizomes could not survive, or if rhizomes subsisted on these sugars, they failed to differentiate. The most widely used sugars in temperate Cymbidium rhizome culture are sucrose and glucose. But their effect on organogenesis and subsequent growth of formed shoots might vary depending on the concentration and type of sugar. Sucrose at $5 \%$ (w) v) was most effective for shoot induction from rhizomes. It was possible to establish the optimum length of time rhizomes required a supply of sucrose by culturing them on hyponex medium in the presence or absence of BA and NAA, and subsequently transferring them from a $5 \%$ sucrose level to a lower sucrose level. The rhizomes on hyponex medium without sucrose did not produce shoots and roots. After 2 weeks of treatment at $5 \%$ sucrose, rhizomes formed shoots and roots following transfer to hyponex medium, which had a lower sucrose level of $2 \%$ or $3 \%$ (Paek et al., 1993). These findings indicate that a high level of exogenous carbohydrate is not required for growth and development during the entire culturc pcriod. We analyzed the changes of sucrose, glucose, fructose, and total free sugar content from rhizome cultures of C. kanran 'Toja' as affected by culture period and growth regulators. In general, regardless of species, visual shoot formation could be observed after 4 or 6 weeks on the shoot-forming medium. The content of sucrose and total free sugars of rhizomes reached maximum at 4 or 6 weeks in culture and, after that, the concentration gradually decreased (Paek et al., 1993). There was no significant difference in total free sugar content between rhizome growth medium and shoot formation medium. In rhizome proliferation media, sucrose concentration reached maximum at 2 weeks in culture and the trend was for decreasing levels with increasing culture age. Nonshoot-forming rhizomes grown on $0.3 \mathrm{mg} \cdot \mathrm{L}^{-1} \mathrm{NAA}-$ containing medium contained more reducing sugars than shoot forming rhizomes.

\section{Conclusions}

De novo organ formation in plants can be induced in many plant species by manipulating the medium and the culture environment. The classical findings of Skoog and Miller (1957) showed that the auxin to cyto- kinin ratio in the medium influenced the pattern of organized development and that shoots could be reproducibly formed in tobacco callus in the presence of a high cytokinin to auxin ratio.

Since the initial culture of explants to first flowering takes a long time (Fig. 2D) and is quite a limiting factor in temperate Cymbidium growth and multiplication, we investigated the physiological and biochemical events during organogenesis and several conditions that controlled morphogenesis by using an in vitro rhizome culture system. Recent research has revealed that the photosynthetic response curves relating to the net photosynthetic rate, photosynthetic photon flux (PPF), and $\mathrm{CO}_{2}$ concentration in the vessel and chamber air temperature were similar to those for tropical Cymbidium plants grown outside and for other $\mathrm{C}_{3}$ plants grown outside under shade (Kozai et al., 1990). These results indicate that $\mathrm{CO}_{2}$ enrichment for the temperatc cymbidiums in vitro at a relatively high PPF could promote photosynthesis and, hence, the growth of chlorophyllous shoots and plantlets in vitro and that the plantlets in vitro would make photoautotrophic growth under environmental conditions favorable to photosynthesis (Kozai, 1990). So far, we do not have much knowledge of organogenesis from rhizomes compared to tobacco systems. In vitro flowering restricted some species that require a long juvenile period before flowering, and segregation of chimera in variegated Cymbidium species as well as chromosomal variations during long-term subculture has posed some problem. So far, not much success has been obtained in this area of research.

\section{Literature cited}

Arditti, J. 1977. Clonal propagation of orchids by means of tissuc culturc-A manual, p. 203-293 In: J. Arditti (ed.). Orchid biology: Reviews and perspectives. Cornell Univ. Press, Ithaca, N.Y.

Arditti, J. and R. Ernst. 1993. Micropropagation of orchids. Wiley, New York.

Chung, J.D., C.K. Chun, S.S. Kim, and J.S. Lee. 1985. Factors affecting growth of rhizomes and organogenesis of Korean native Cymbidium kanran. Kor. Soc. Hort. Sci. 26:281-288.

Hasegawa, A. and M. Goi. 1987. Rhizome formation in Cymbidium goeringii Reichenbach fil and Cymbidium kanran Makino in shoot tip culture. Jpn. Soc. Hort. Sci. 56:70-78.

Ishii, M. 1974. Partial elimination of virus from doubly infected orchids by meristem explant culture. Acta Hort. 36:229-233.

Knudson, L. 1946. A nutrient for the germination of orchid seeds. Amer. Orchid Soc. Bul. 15:214217. 
Kozai, T., H. Oki, and K. Fujiwara. 1990. Photosynthetic characteristics of Cymbidium plantlets in vitro. Plant Cell Tissue Organ Cult. 22:205-211.

Kozai, T. 1990. Micropropagation under photoautotrophic conditions, p. 447-469 In: P.C. Debergh and R.H. Zimmerman (eds.). Micropropagation: Technology and application. Kluwer Academic Publishers, Boston.

Lee, J.S., K.K. Shim, M.S. Yoo, J.S. Lee, and Y.J. Kim. 1986. Studies on rhizome growth and organogenesis of Cymbidium kanran cultured in vitro. Kor. Soc. Hort. Sci. 27:174-180.

Morel, G.M. 1960. Producing virus-free Cymbidiums. Amer. Orchid Soc. Bul. 29:495-497.

Murashige T. and F. Skoog. 1962. A revised medium for rapid growth and bioassays with tobacco cultures. Physiol. Plant. 15:473-497.

Paek, K.Y., G.B. Shim, and J.K. Whang. 1987 Asymbiotic germination and plantlet of rhizome through the aseptic culture of temperate Cymbidium seeds. Kor. Soc. Hort. Sci. 28:185-193.

Paek, K.Y., G.B. Shim, and J.J. Kim. 1989. Asymbiotic germination of temperate cymbidiums and effect of media and growth regulators on organogenesis. Kor. Soc. Hort. Sci. 30:234-247.

Paek, K.Y., G.B. Shim, and J.J. Kim. 1990. Effect of natural products and BAP exposing periods on organogenesis of temperate cymbidiums using rhizomes. Kor. Soc. Hort. Sci. 31:74-80.

Pack, K.Y., S.Y. Ahn, and G.B. Shim. 1990a. Anatomical observations on organogenesis and polyphenol content, isoenzyme analysis of in vitro cultured rhizomes in temperate cymbidiums. Kor. Soc. Hort. Sci. 31:263-275.
Paek, K.Y., G.B. Shim, C.H. Lee, J.J. Kim, and K.W. Kang. 1990b. Micropropagation and in vitro breeding of tempcrate Cymbidium, p. 231237. Proc. 13th World Orchid Conf. 1991, Auckland, N.Z.

Pack, K.Y. and E.C. Yeung. 1991. The effect of 1naphthalene acetic acid and $\mathrm{N}^{6}$-Benzyladenine on the growth of Cymbidium forrestii rhizome in vitro. Plant Cell Tissue Organ Cult. 24:65-71.

Paek, K.Y., G.J. Yoo, M.R. Park, and S. Hisajima. 1993. Effect of sucrose gradient and changes of free sugar content during organogenesis through rhizome culture of temperate Cymbidium, p. 5563. Proc. Nagoya Intl. Orchid Conf. Nagoya.

Shimasaki, K. and S. Uemoto. 1990. Micropropagation of a terrestrial Cymbidium species using rhizomes developed from seeds and pseudobulbs. Plant Cell Tissue Organ Cult. 22:237-244.

Skoog, F. and C.O. Miller. 1957. Chemical regulation of growth and organ formation. Expt. Biol. 11:118-131.

Ueda, H. and H. Torikata. 1969. Organogenesis in the meristem cultures of cymbidiums. Histological studies on the shoot formation at rhizometips of Cymbidium goeringii Reichb. f. cultured in vitro. Jap. Soc. Hort. Sci. 38:262-266.

Wang, X., J. Zhang., H. Lian, and S. Gong. 1988. Studies on Cymbidium ensifolium Susin clonal propagation and floral differentiation by means of tissue culture. Acta. Hort. Sinica 15:205-208

Wu, H., X. Wang, and T. Lin. 1987. A study on the shoot tip culture of oriental orchid. Acta. Hort. Sinica 14:203-207. 